\title{
GOBERNANZA LOCAL Y ESPACIO RURAL: UN ANÁLISIS TERRITORIAL DESDE LA PERSPECTIVA DE GÉNERO'
}

\author{
Antònia Casellas \\ Antoni F. Tulla \\ Ana Vera \\ Marta Pallarès Blanch \\ Department de Geografia. Universitat. Autònoma de Barcelona \\ antonia.casellas@uab.cat, antoni.tulla@uab.cat, ana.vera@uab.cat \\ marta.pallares@gmail.com
}

\section{RESUMEN:}

Desde una perspectiva de género el presente artículo analiza el nivel y características de la participación en política local de las mujeres dentro del ámbito rural. El estudio asume que la gobernanza territorial debe incluir no sólo aspectos económicos y de gestión, sino también de participación política, tanto directa como indirectamente. A través del caso de estudio de seis comarcas del Pirineo catalán, combinando una metodología cuantitativa y cualitativa, se analizan datos electorales y se identifican componentes de oferta y demanda para determinar que factores facilitan y cuales dificultan la participación activa de las mujeres en la política municipal.

Palabras clave: Mujeres, gobernanza, rural, participación política, género.

Fecha de recepción: diciembre 2011.

Fecha de aceptación: enero 2013.

1 Esta investigación ha tenido el apoyo del Institut Català de les Dones (ICDs) de la Generalitat de Catalunya (Exp U-9/10), el contrato de investigación Ramón y Cajal (RYC-2008-02456) y el proyecto del MICIIN CSO200908271. La contribución de Pallarès Blanch se inscribe en el marco de su tesis doctoral. Los autores desean agradecer las contribuciones de dos revisores quienes han contribuido con sus sugerencias a la mejora del artículo. 


\section{ABSTRACT:}

This paper analyses the level and features of women's local political participation in rural areas. The study assumes that territorial governance must include not only economic and management aspects, but also political participation, both directly and indirectly. Through a case study of six counties in the Catalan Pyrenees, combining quantitative and qualitative methodology, we analyse electoral data and identify supply and demand factors to establish which factors facilitate and which constrain the active involvement of rural women in local politics.

Key words: Women, governance, rural, political participation, gender.

\section{INTRODUCCIÓN}

Las zonas de montaña son a menudo sinónimo de espacios conservadores donde las estructuras de poder y las costumbres sociales cambian lentamente. Un buen indicador de progreso de estas zonas es el grado de participación de las mujeres en las decisiones públicas. Una amplia gama de pensadores de diferentes disciplinas han enfatizado el hecho de que en numerosas culturas y estadios de desarrollo las mujeres han estado históricamente subordinadas a los hombres (Malinowski, 1955; Wollstonecraft, 1975; Benería y Sen, 1982; Harley, 2007). Desde el inicio de la democracia en España, las mujeres han mejorado considerablemente su situación económica, política y social, pero con todo, siguen estando en situación de desventaja respecto a los hombres, aspecto que se hace aún más evidente en las zonas rurales.

Un estudio de diagnóstico de la igualdad de género en el mundo rural en España elaborado por el Ministerio de Medio Ambiente, Rural y Marino en el 2011 revela, a partir de una encuesta representativa a 4.500 habitantes de entre 20 y 65 años, que un $25 \%$ de los habitantes de los pueblos de España cree que hombres y mujeres no pueden realizar las mismas funciones, ni tener las mismas responsabilidades. Un 30\% de esta población cree que las mujeres deben sacrificar su faceta productiva en favor de la reproductiva, y conservar la tradicional atribución de roles. Estos datos demuestran que los procesos de desigualdad persisten y que, como señalan algunos estudios (Binimelis et al,2008), se produce en el caso de las mujeres del ámbito rural una doble limitación, por ser mujeres y por vivir en zonas rurales, donde se perpetua una estructura más patriarcal y una falta de servicios y oportunidades, en comparación a las que pueden encontrarse en las ciudades y zonas más urbanizadas. Se evidencia pues la necesidad de nuevos estudios y propuestas que profundicen en la comprensión de las dinámicas de género en el ámbito rural.

Con este objetivo, el presente artículo analiza el nivel de participación política de las mujeres en la escala local rural, a través del caso de estudio de la presencia de mujeres en la política municipal en seis comarcas del Pirineo catalán. El estudio investiga el grado de participación y determina los factores que facilitan o limitan la intervención activa de las mujeres en los consistorios municipales en calidad de alcaldesas y regidoras. Con este fin, el siguiente apartado presenta el enfoque analítico en el que se enmarca la investigación, intro- 
duciendo el concepto de gobernanza como mecanismo de gestión, no tan sólo de la estructura administrativa y económica, sino también de los procesos políticos y sociales de un espacio (Farinós, 2008). A continuación se describe el marco orográfico y socioeconómico del área de estudio, el cual ejemplifica la relevancia del componente geográfico e histórico del territorio. En las siguientes secciones se detalla la metodología utilizada para el análisis y se realiza el análisis temporal del grado de participación de las mujeres en España y Cataluña, para después evaluar el caso de las seis comarcas de montaña. Una vez determinada la evolución y nivel de participación de las mujeres en la política municipal de los casos de estudio, en el siguiente apartado se identifican elementos sociales, culturales y políticos que limitan o facilitan la participación activa de las mujeres en la política local a través del análisis temático de treinta entrevistas semi-estructuradas. El artículo concluye con unas reflexiones finales que aportan claves de actuación y análisis, tanto para las administraciones públicas como para los estudios de género y del mundo rural.

\section{MUJERES Y GOBERNANZA COMO ÁMBITO DE ANÁLISIS}

La definición de gobernanza aprobada por el Programa de Desarrollo de las Naciones Unidas en el año 2000 define la gobernanza como el ejercicio de la política, la economía y la autoridad administrativa para gestionar los asuntos de un país y, por ello se compone de los mecanismos, procesos e instituciones a través de los cuales los ciudadanos y los grupos de la sociedad civil articulan sus intereses, ejercen sus derechos legales, cumplen sus obligaciones y median sus diferencias (UNDP, 2003). Se entiende por tanto que la gobernanza, en sentido amplio, incluye una amplia gama de formas, en las que la política y la estructura social y administrativa de una sociedad afectan el acceso de sus miembros a las oportunidades básicas y al desarrollo de sus capacidades. Entendida de esta forma, la gobernanza territorial debe pues incluir no sólo aspectos económicos y de gestión, sino también la participación política, tanto a través de la participación en instituciones formales incluyendo medidas legislativas, jurídicas, administrativas e instituciones, como de forma informal, en movimientos e instituciones de la sociedad civil (Farinós, 2008).

En relación a la esfera política, en los últimos años las mujeres españolas han sido designadas para cargos políticos de forma creciente. En marzo del 2007, la Ley Orgánica 3/2007 para la igualdad efectiva de mujeres y hombres introducía la obligatoriedad para los partidos políticos de confeccionar candidaturas en las que las mujeres deberían estar representadas en al menos un $40 \%$ en las listas electorales, en tramos de cinco puestos, salvo en las localidades de menos de 5.000 habitantes -menos de 3.000 habitantes a partir del 2011. A pesar de este avance en materia legislativa, la realidad política de las mujeres en general en España (Verge, 2010), y de las comarcas del Pirineo en particular (Casellas, at al, 2009), se encuentra lejos de ser equiparable a la de los hombres.

En el Pirineo, la doble discriminación que sufren las mujeres en términos de producción y reproducción se ve acentuada por las condiciones geográficas, la falta de opciones de empleo y la menor capacidad de los gobiernos locales para prestar servicios a las mujeres en concreto, y a los ciudadanos en general (Tulla, 1991). Como se analizará más adelante, estas zonas rurales están menos densamente pobladas, y por tanto tienen una menor capacidad para recaudar impuestos y pagar servicios. Asimismo, el aislamiento de las zonas de montaña 
ha ayudado a preservar las estructuras patriarcales y tradicionales (Binimelis et al, 2008), generando limitadas oportunidades de empleo (García-Ramón, et al. 1995; Monk y Hodge, 1995; Viladomiu y Rosell, 1998).

El limitado papel de las mujeres en política rural ha sido identificado en estudios de carácter internacional. De entre ellos destaca el trabajo de Massolo (2003), quien encabezó un estudio sobre las localidades rurales y los centros urbanos intermedios de diversos países latinoamericanos mostrando que tan solo un 5,3\% de los consistorios estaban liderados por mujeres alcaldesas. Un estudio realizado en Brasil muestra un porcentaje también limitado de participación femenina, ya que las mujeres representan el 9\% de miembros de los ayuntamientos en municipios del Estado de San Pablo (Brabo, 2008). En trabajos latinoamericanos se identifica que la participación activa en movimientos sociales es una condición presente en gran número de mujeres que posteriormente forman parte de las listas electorales municipales (Silva, 2011). En Europa una parte de investigaciones sobre la participación de las mujeres en los gobiernos locales, como por ejemplo en Hungría (Timar, 2004), se han centrado en los municipios urbanos. En España se han realizado estudios sobre las mujeres empresarias y cargos electos en municipios rurales grandes de Andalucía (Palenzuelo Chamorro, 2002). Más frecuentes han sido los trabajos que tratan de la presencia de la mujer en las comunidades autónomas, tanto en calidad de políticas y como de técnicas. Se ha debatido ampliamente sobre la incorporación de la mujer en la política formal como mera labor continuista de la desarrollada por los hombres o, por el contrario, como ejemplo de una ruptura en los modelos de hacer política (Otero, 2006).

Los estudios especializados en identificar los factores que intervienen en la participación de las mujeres en política distinguen entre los factores de demanda y los factores de oferta (Norris, 1997). A grandes rasgos, los factores de demanda vienen representados por el grado de necesidad o de capacidad de integración que hay para incluir a mujeres en los órganos de representación política. En este sentido la ley aprobada en el 2007 supone un gran paso adelante, aunque limitado con relación a los municipios de montaña, ya que, en principio, sólo afecta a aquellas poblaciones de hasta 3.000 o más habitantes a partir del 2011. Los factores de oferta que afectan el nivel de inclusión de las mujeres en la política incluyen el grado de interés o predisposición que las mujeres sienten o tienen para ofrecerse como candidatas a los órganos de representación.

Tanto los factores de oferta como los de demanda están condicionados por los valores culturales dominantes, que implican una visión determinada de la sociedad, ligada y vinculada de manera muy especial en zonas de montaña, a representaciones estereotipadas según el sexo de las personas. Así los estereotipos de género juegan un triple papel, operan en las mentalidades de los cargos internos de los partidos, operan en las mentalidades de las propias mujeres y operan a nivel del conjunto de la sociedad. En las representaciones subjetivas que condicionan la inclusión de las mujeres en los órganos de gobierno hay que añadir los apremios que viven las mujeres en su día a día como producto del rol que, de una forma aún bastante generalizada, asigna una distribución de tareas entre sexos asimétrica en muchos niveles. Este fenómeno se define a través de la expresión doble jornada, la del trabajo remunerado y la del trabajo doméstico, y triple jornada si se suman las funciones de socialización del núcleo familiar y la participación en redes de relación. Las limitaciones culturales relacionadas con los papeles del rol de género llevan a lo que se identifica como techo de cristal, 
una mayor dificultad de las mujeres para alcanzar los puestos de mayor responsabilidad, resultado de la suma de los diferentes impedimentos tangibles e intangibles que obstaculizarían la inclusión de las mujeres en los órganos de representación política, como también sucede en los cargos de dirección de las empresas o de la universidad (Valcárcel 1997; Pujol y Ortiz, 2009).

\section{III. ÁREA DE ESTUDIO Y METODOLOGÍA}

\section{Características orográficas y socioeconómicas del caso de estudio}

La región del Alt Pirineu i Aran (APIA) se localiza al noroeste de Cataluña. La forman las comarcas del Alt Urgell, el Alta Ribagorça, la Cerdanya, el Pallars Jussà, el Pallars Sobirà y la Val d'Aran. En total las 6 comarcas ocupan una superficie de $5.686 \mathrm{~km}^{2}$, el 17,8\% de Cataluña, y tienen una población de 76.662 habitantes en el año 2010, la cual representa el $1,02 \%$ del total de la población de Cataluña.

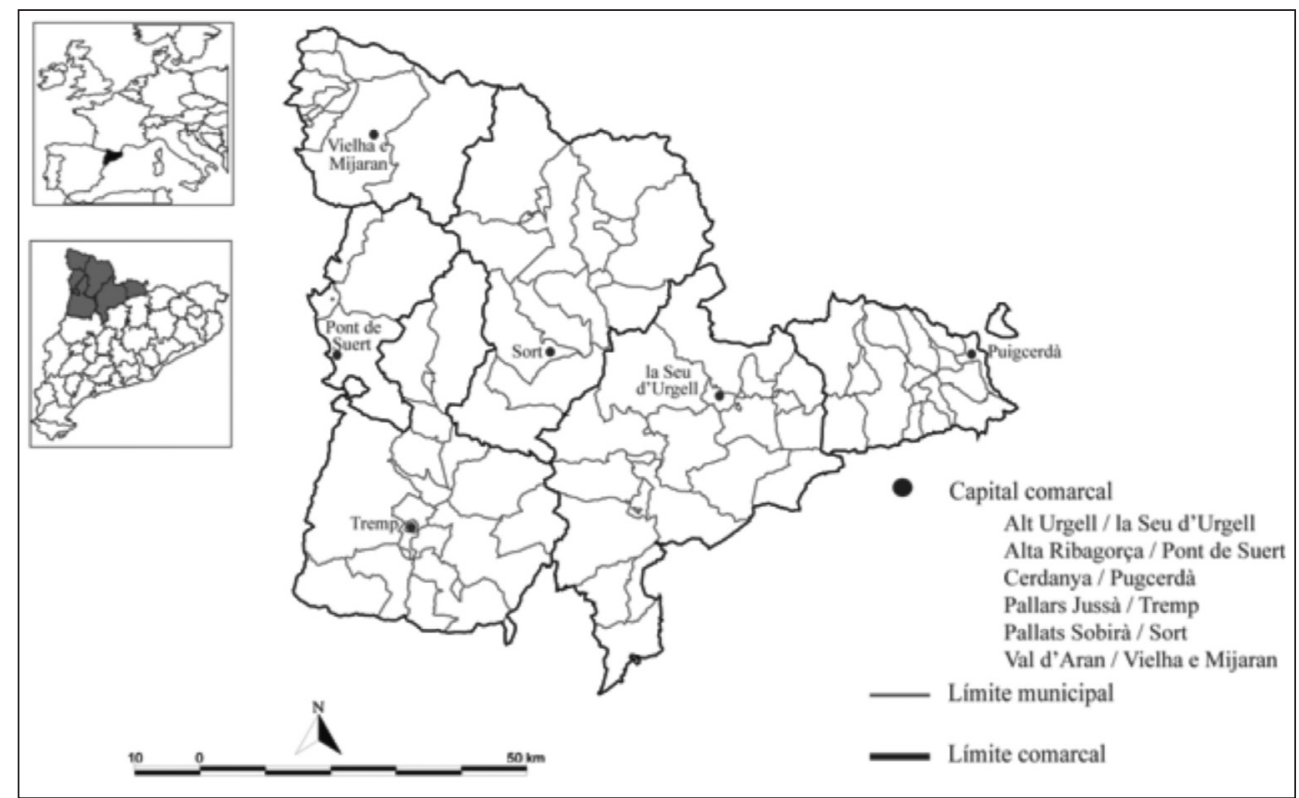

Fuente: Elaboración propia a partir de las Bases Cartográficas del Departament de Territori i Sostenibilitat de la Generalitat de Catalunya, último acceso 25-05-2012.

Para contextualizar el ámbito político y el grado de participación de las mujeres en los municipios de esas comarcas es necesario prestar atención al desarrollo socioeconómico que ha tenido el APIA a lo largo de todo el siglo XX e inicio del XXI. El primer aspecto a resaltar es la estructura orográfica, la cual ha condicionado el desarrollo del espacio pro- 
ductivo, el aprovechamiento de los recursos naturales y la accesibilidad. El APIA presenta unas condiciones orográficas únicas que se caracterizan por una orientación este-oeste de la cordillera pirenaica marcada por una disimetría entre umbrías y solanas, y un eje norte-sur de un carácter fluvial muy acentuado (Figura 2). Esta morfología ha beneficiado o limitado los diferentes sectores económicos de la zona. El sector primario se ha centrado en los cultivos, la ganadería de vacunos y el aprovechamiento forestal. El sector secundario se ha especializado en la extracción del agua como fuente de energía hidroeléctrica y la explotación de la madera. Indirectamente, el turismo como sector terciario, y la construcción han utilizado la morfología y el paisaje que les rodea para desarrollar su actividad económica.

Un segundo aspecto a tener en cuenta para el análisis de la gobernanza local es el proceso de despoblamiento y poblamiento que se ha dado a lo largo de los años. La tendencia general en el Pirineo catalán hasta finales del siglo XX ha sido la disminución progresiva de población causada principalmente por la pérdida de puestos de trabajo. La recuperación económica de principios del siglo XXI, y por lo tanto, la necesidad de mano de obra ha llevado a una repoblación significativa de la zona a partir del año 2000. Finalmente, en tercer lugar, y de forma muy vinculada con el punto anterior, cabe enfatizar la relación de masculinidad histórica que predomina en el conjunto del territorio y que condiciona y ha condicionado los puestos de trabajo y el desarrollo económico local del área.

Figura 2

DISTRIBUCIÓN DE RECURSOS NATURALES Y ESPACIOS URBANOS

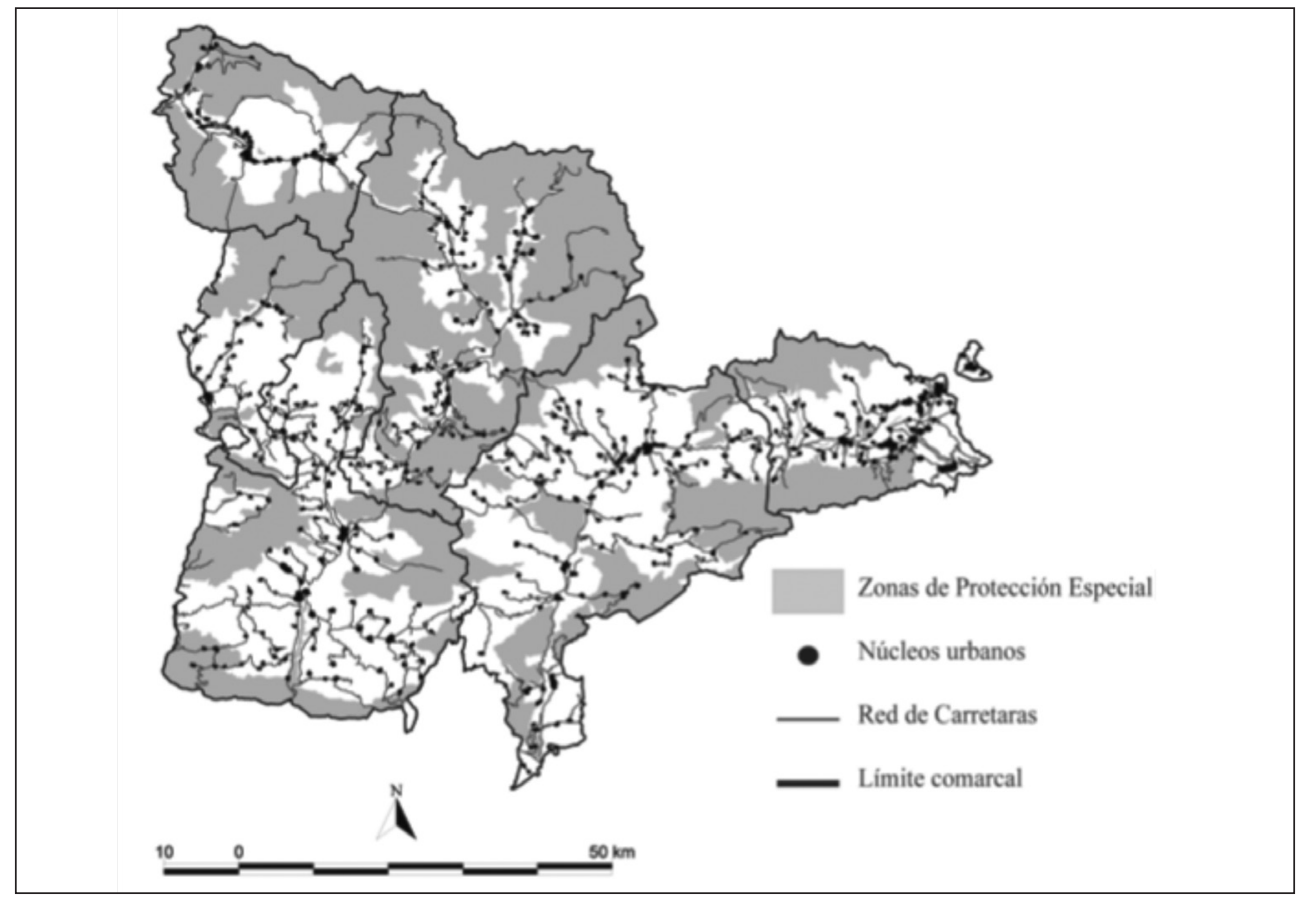

Fuente: Elaboración propia a partir de las Bases Cartográficas del Departament de Territori i Sostenibilitat de la Generalitat de Catalunya, último acceso 25-05-2012. 
A lo largo de más de un siglo, entre 1900 y 2010 se han alternado períodos con características migratorias diferentes. La primera oleada migratoria significativa en esta zona se remonta al período de la Guerra Civil (1936-1939) durante la cual se despoblaron núcleos urbanos enteros. Posteriormente, en los años cincuenta del siglo XX se produce una recuperación económica y demográfica directamente relacionada con la construcción de los embalses y centrales hidroeléctricas. Este factor se ha observado especialmente en las comarcas de la Alta Ribagorça, Pallars Jussà, Pallars Sobirà y Vall d'Aran. En este periodo también hay un proceso de redistribución de la población interna, de forma que son las capitales comarcales como Puigcerdà en la Cerdanya o la Seu d'Urgell en el Alt Urgell las que aumentan significativamente en número de habitantes como consecuencia de la migración proveniente de municipios situados a mayor altitud (Figura 1). Es en el año 1991 cuando el Pirineo llega a un mínimo de población -59.382 habitantes- 0,94\% de Cataluña (IDESCAT, 2011a). Este descenso demográfico se produce como resultado de las dificultades para la tecnificación y la innovación en las explotaciones agrarias tradicionales y la existencia de desigualdades para poder acceder a servicios básicos. Ello comporta un abandono de los pueblos y un envejecimiento marcado de la población (Guirado, 2011). Este proceso inicia una tendencia reversible en los años ochenta, cuando se observan pequeños movimientos migratorios de grupos neorurales hacia la zona (Soriano y Tulla, 2003; Nogué, 1988). A partir de los noventa hasta el 2010 hay un cambio en positivo en la tendencia migratoria basada en la llegada de población vinculada a la oferta de trabajo en la actividad turística y en la construcción. Esta transformación económica y demográfica representará beneficios, pero también supondrá una importante presión sobre el medio, la vivienda y los servicios (Vilagrasa, 2003).

La evolución de la población activa de la zona entre el 2000 y 2010 varía dependiendo del sexo. En el contexto de la crisis económica iniciada en el año 2007 se observa que se ha producido una disminución sensible del porcentaje de población activa para los hombres, pasando del $74,58 \%$ en el 2000 al $72,64 \%$ en el 2010 , mientras que la población activa de las mujeres en el mismo período se ha mantenido prácticamente constante, del 70,61\% al $70,08 \%$ respectivamente. Con respecto a Cataluña la población activa se ha mantenido por encima de la del APIA, aunque también ha disminuido. Los hombres han pasado de un $75,95 \%$ a un $73,77 \%$ desde 2000 a 2010 , y las mujeres de un $72,72 \%$ a un $70,59 \%$. La población ocupada y los valores del PIB indican que la estructura económica del APIA está especializada en el sector de los servicios, prestando especial atención a los subsectores de la hostelería, el comercio y la reparación, los servicios a las empresas y la construcción. En valores porcentuales, la población ocupada en el sector servicios es de un 66,2\% en el APIA frente a un $63,5 \%$ para el total de Cataluña. La construcción ha representado en esta zona un $18,5 \%$, muy por encima de Cataluña con un 10,09\% (IDESCAT, 2011b).

El análisis de las diferencias en el empleo entre los hombres y las mujeres muestra la misma tendencia que para el conjunto de Cataluña. La distribución de actividad por sexos indica que en el sector servicios, la población femenina del APIA tiene una mayor participación, con un $90 \%$ de ocupación, mientras que en los otros sectores es muy baja. En cuanto a la población masculina también es importante su participación en el sector servicios, pero por debajo de los valores de Cataluña, 48,6\% frente 51,2\%. También es significativa la población ocupada en la agrupación de sectores industria y construcción, donde se da un 42,9\% en el 
APIA y un 45,9\% para Cataluña. Si analizamos los valores a nivel comarcal se observa que son las comarcas de la Vall d'Aran y la Cerdanya las que concentran más población ocupada en la construcción. También se identifica que la participación de las mujeres en el sector agrario se concentra en el Alt Urgell, el Pallars Jussà y la Cerdanya. Por profesión y sexo, en el ámbito de población ocupada hay una distribución desigual por sexos, destacando el predominio de las mujeres en las categorías de: técnicos y profesionales científicos intelectuales $(58,27 \%)$, empleados administrativos $(60,73 \%)$ y trabajadores de servicios y vendedores de comercio $(63,97 \%)$; coincidiendo con los subsectores de administración pública, sanidad y enseñanza. Los hombres predominan con un $61,13 \%$ en la categoría de personal directivo de las empresas y administraciones públicas (IDESCAT, 2011b).

\section{Metodología}

Para el presente estudio se ha utilizado una combinación de métodos, incluyendo técnicas cualitativas y cuantitativas. En primer lugar se ha realizado una investigación documental sobre mujeres y participación política para determinar las líneas de análisis. Asimismo se ha realizado un análisis de indicadores socioeconómicos y demográficos para contextualizar las comarcas analizadas, ampliando esta información con datos orográficos. Respecto a la valoración de la participación de las mujeres en política, en diferentes escalas espaciales, se ha trabajado con la base de datos de diferentes legislaturas, desde la primera legislatura de 1979-83 hasta la de 2007-11, con el fin de aportar y analizar la participación de las mujeres en el ámbito español, catalán y municipal con base temporal.

El trabajo de campo ha comportado la realización de 30 entrevistas, que como la tabla 1 específica se distribuyen por comarcas, individuos con experiencia política, técnica y sexo. Las entrevistas semi-estructuradas en un guion original previo abordaban los siguientes aspectos: factores facilitadores y limitadores de la participación política de las mujeres; características y factores en la elaboración de cuadros y listas electorales; características de los cargos públicos ostentados y valoración de la influencia dentro del ámbito local; valoraciones de la actividad política; y temas relevantes entre representantes públicos y sociedad civil rural. Las entrevistas se realizaron durante los meses de julio a octubre de 2011 en los municipios donde ejercen la gestión los entrevistados/as. En el análisis de las entrevistas se ha optado por la utilización del análisis temático (Leininger, 1985; Taylor y Board, 1984; Spradley, 1979). La transcripción de las entrevistas ha sido realizada utilizando el sistema verbatim, que consiste en la transcripción de toda la información grabada, incluyendo las palabras tal y como se han dicho, al igual que coletillas, repeticiones, pausas, risas, etc. Este tipo de análisis permite identificar de entre los datos los elementos relevantes a los ejes de investigación.

El análisis de esta parte de la investigación se centra en identificar los factores facilitadores y limitadores que condicionan la participación de las mujeres en la política local, aportando datos cualitativos que hacen visibles aspectos difíciles de identificar a través de otro tipo de investigaciones. En el proceso de estudio del material se han realizado varios niveles de análisis. En primer lugar, con las conversaciones transcritas se han identificado patrones de experiencias a través de citas directas, algunas de las cuales han sido reproducidas para ilustrar este trabajo. El siguiente paso ha consistido en identificar todos los datos 
Tabla 1

DISTRIBUCIÓN DE ENTREVISTAS EN PROFUNDIDAD REALIZADAS EN EL TRABAJO DE CAMPO

\begin{tabular}{|l|c|c|c|c|c|}
\hline \multirow{2}{*}{ Comarca } & \multicolumn{2}{|c|}{ Representantes políticos } & \multicolumn{2}{c|}{ Técnicos y especialistas } & \multirow{2}{*}{ Total } \\
\cline { 2 - 5 } & Mujeres & Hombres & Mujeres & Hombres & \\
\hline Alt Urgell & 6 & 3 & & & 9 \\
\hline Alta Ribagorça & 1 & 1 & & & 2 \\
\hline Cerdanya & 1 & 2 & & & 3 \\
\hline Pallars Jussà & 1 & 1 & & & 2 \\
\hline Pallars Sobirà & 3 & 2 & & & 5 \\
\hline Val d'Aran & 1 & 1 & 1 & & 3 \\
\hline Alt Pirineu i Aran & & & 4 & 2 & 6 \\
\hline \multicolumn{1}{|c|}{ Total } & 13 & 10 & 5 & 2 & 30 \\
\hline
\end{tabular}

Fuente: Elaboración propia a partir del trabajo de campo.

de las entrevistas referidos a los patrones ya clasificados, para continuar con la creación de subtemas resultantes de la combinación de patrones. A continuación se han definido las unidades temáticas. Estas unidades temáticas incluyen temas de conversación, vocabulario, actividades recurrentes, significados o sentimientos (Taylor y Board, 1984). Finalmente se han identificado los temas reuniendo los componentes o fragmentos de ideas y experiencias. De esta forma los temas han surgido de las historias de los informantes, las cuales se han ensamblado para formar una imagen completa de su experiencia colectiva (Leininger, 1985). En el apartado quinto se presenta este análisis y se aportan citas directas que se identifican con la letra «E» y un dígito, correspondiente al número de la entrevista, y la inicial «M» $\mathrm{O}$ «H», la cual permite identificar si el entrevistado es hombre o mujer. La última letra en la identificación, «P»o $\ll \mathrm{T} »$, indica si el entrevistado es político/a o técnico/a.

\section{GRADO Y CONDICIONANTES DE LA INTERVENCIÓN DE LAS MUJERES EN POLÍTICA}

\section{El contexto español y catalán}

Para contextualizar la participación y actividad política de las mujeres en las comarcas de montaña del Pirineo catalán y analizar su evolución, en primer lugar examinamos la participación política femenina en el ámbito español. En su conjunto, la presencia de mujeres a nivel del parlamento español ha ido evolucionando positivamente del 6,7\% en 1987 a 41,1\% en 2007, vinculado a la aplicación del nuevo marco legislativo (Verge, 2010). En cuanto a la escala de comunidades autonómicas, en 1987 hubo diferencias importantes entre los niveles bajos de ciertas comunidades, como Murcia (0\%), Galicia (1,4\%) y Aragón (1,5\%), respecto a otras comunidades con parlamentos con una mayor presencia de mujeres, como Madrid $(16,7 \%)$ y País Vasco $(13,3 \%)$. Un hecho a destacar es el caso de Cataluña, que como muestra la tabla 2 , se sitúa muy por debajo de estas dos comunidades. 
Tabla 2

EVOLUCIÓN DE LA PRESENCIA DE LAS MUJERES EN LOS PARLAMENTOS AUTONÓMICOS

\begin{tabular}{|l|c|c|c|c|c|c|}
\hline \multirow{2}{*}{ Comunidades Autónomas } & \multicolumn{2}{|c|}{1987} & \multicolumn{2}{c|}{1997} & \multicolumn{2}{c|}{2007} \\
\cline { 2 - 7 } & Total (N) & Mujeres \% & Total (N) & Mujeres \% & Total (N) & Mujeres \% \\
\hline Andalucía & 109 & 4,6 & 109 & 28,4 & 109 & 39,4 \\
\hline Aragón & 67 & 1,5 & 67 & 9,0 & 67 & 35,8 \\
\hline Asturias & 45 & 11,1 & 45 & 20,0 & 45 & 33,3 \\
\hline Islas Balears & 59 & 6,8 & 59 & 30,5 & 59 & 49,2 \\
\hline Islas Canarias & 60 & 1,7 & 60 & 13,3 & 60 & 40,0 \\
\hline Cantabria & 39 & 7,7 & 39 & 15,4 & 42 & 40,5 \\
\hline Castilla-León & 84 & 3,6 & 84 & 20,2 & 83 & 43,4 \\
\hline Castilla-La Mancha & 42 & 9,5 & 47 & 23,4 & 47 & 53,2 \\
\hline Cataluña & 135 & 8,9 & 135 & 14,8 & 135 & 36,3 \\
\hline Extremadura & 64 & 3,1 & 65 & 16,9 & 65 & 40,0 \\
\hline Galicia & 71 & 1,4 & 78 & 16,7 & 75 & 33,3 \\
\hline Madrid & 96 & 16,7 & 103 & 27,2 & 120 & 42,5 \\
\hline Murcia & 45 & 0,0 & 45 & 15,6 & 46 & 39,1 \\
\hline Navarra & 50 & 8,0 & 50 & 18,0 & 50 & 38,0 \\
\hline La Rioja & 33 & 9,1 & 33 & 21,2 & 33 & 39,4 \\
\hline Valencia & - & - & - & - & 25 & 40,0 \\
\hline País Vasco & 90 & 5,6 & 89 & 24,7 & 99 & 45,5 \\
\hline Ceuta & 1.164 & 13,3 & 75 & 24,0 & 75 & 52,0 \\
\hline Melilla & - & - & - & 25 & 40,0 \\
\hline Total (\%) & & -7 & 1.183 & & 1.260 & \\
\hline Total (N) & & & $-1,4$ & \\
\hline
\end{tabular}

Nota: $\mathrm{N}=$ total Hombres y Mujeres

Fuente: Elaboración a partir de datos de Instituto de la Mujer, 2010a.

Aunque Cataluña, al igual que Madrid y el País Vasco es una de las regiones económicamente más prósperas de España, en 1987 la comunidad catalana presentaba un menor porcentaje de mujeres, con un $8,9 \%$, sólo unas pocas décimas por encima de la media española. Veinte años más tarde, en 2007, y como resultado de la nueva legislación aprobada en 2007 que estableció una presencia mínima de mujeres en las listas electorales, la presencia media de mujeres legisladoras en los gobiernos autonómicos aumentó a un notable 41\%. En este nuevo contexto, Cataluña aún presenta un sorprendentemente bajo porcentaje, un 36,3\%, después de haber perdido terreno con respecto al porcentaje medio español y encontrarse 


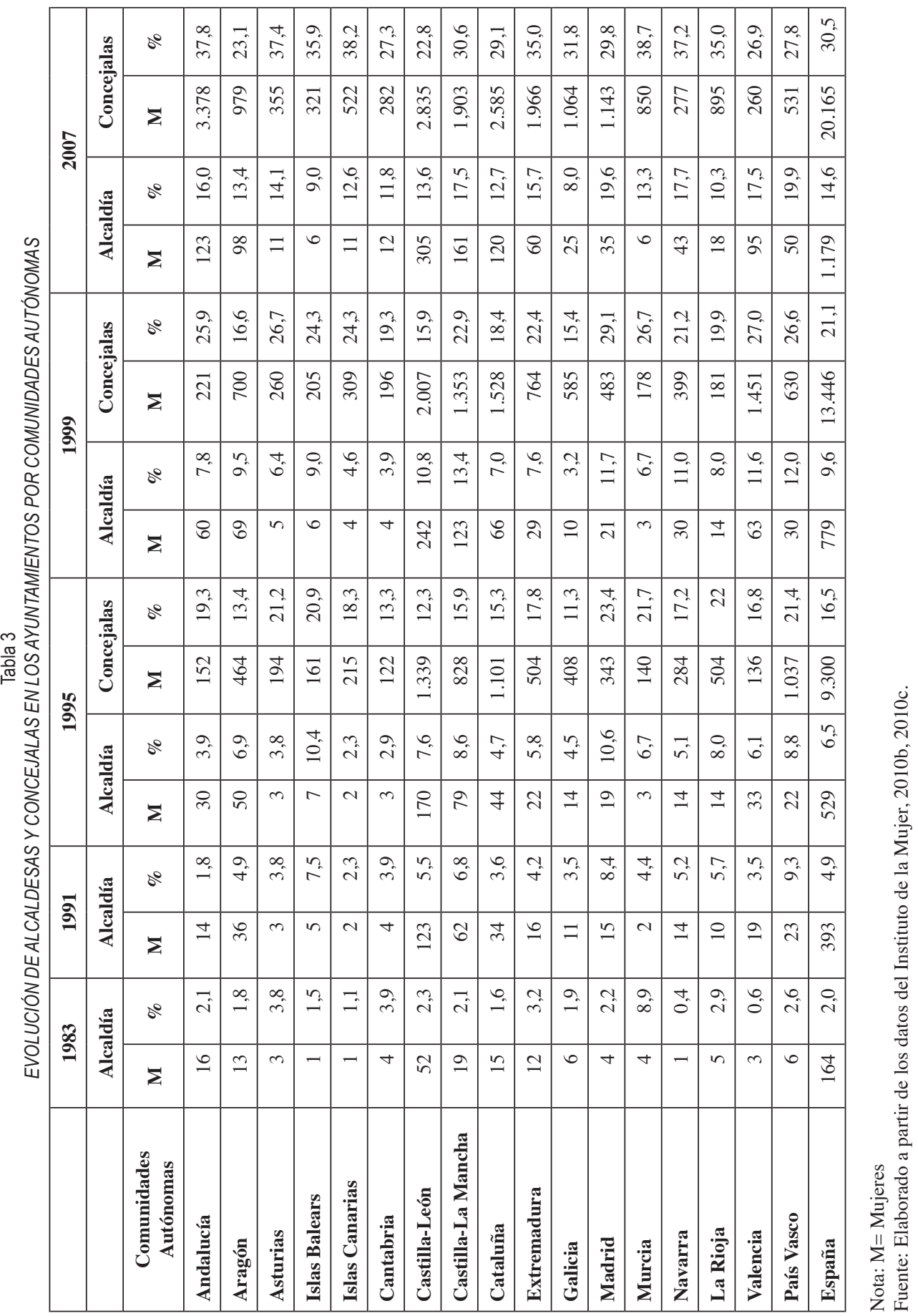


por debajo de quince comunidades autonómicas, de tal forma que sólo Aragón, Asturias y Extremadura cuentan con un número inferior de mujeres en sus parlamentos autonómicos.

Si nos centramos en la participación de las mujeres en los gobiernos ejecutivos autonómicos, el resultado global para España es también un aumento de la presencia femenina desde el $11 \%$ en 1996, a un $40 \%$ de mujeres en ejecutivos de los gobiernos regionales para el año 2009. Los mayores porcentajes de presencia de mujeres se encuentran en Extremadura (62\%) y Andalucía (60\%). En cuanto a Cataluña, la comunidad mejora respecto al ámbito legislativo, ya que la presencia de mujeres en el ejecutivo en el 2009 es de un 58\%. En este sentido, Cataluña se sitúa significativamente por encima de la media española del $40 \%$.

Respecto a la escala municipal, el número de mujeres alcaldesas en 1983 fue de 164 para el conjunto de España, lo que representa un $2 \%$ de los alcaldes en una totalidad de más de 8.000 municipios (tabla 3). En 2007, el número de alcaldesas aumentó a un 14,6\% o un total de 1.179 mujeres alcaldesas en todo el territorio nacional. En cuanto a los miembros concejales, los datos de 1995 a 2007 en los municipios españoles revelan que ha habido un incremento del $16,5 \%$ al 30,5\% de mujeres concejales. Dentro de esta situación, Cataluña, aunque ha aumentado el número de mujeres alcaldesas, ha permanecido por debajo de la media española durante todo el periodo, pasando del 1,6\% con respecto a la media española del $2 \%$ en 1983, al 12,7\% de 2007, siendo el 14,6\% la media del país. Los datos sobre la composición de las concejalías también revelan que Cataluña ha presentado en nivel constantemente inferior a los valores de la media de España.

Como valoración general se puede afirmar que en el análisis de la representación institucional de las mujeres en Cataluña es sorprendente que, aunque esta es una región fuertemente urbanizada, con una larga tradición industrial, así como una historia de amplios movimientos sociales, la presencia de mujeres en la política y en puestos de responsabilidad es significativamente menor que el promedio de España.

\section{La participación política de las mujeres en el Pirineo catalán}

Las comarcas de montaña de Cataluña, especialmente las del APIA tienen un reto específico debido a las características geográficas, demográficas y económicas tal como se ha descrito en el apartado anterior. Históricamente ha habido una mayor persistencia del sistema de subsistencia agraria, que más tarde ha evolucionado hacia la progresiva producción terciaria, con escaso desarrollo industrial (Tulla, 1991). Esto explicaría en gran medida la debilidad de la infraestructura urbana de los Pirineos, no sólo en términos del tamaño de los centros de población, sino también, en cuanto a la complejidad urbana. En el análisis de los representantes elegidos en los gobiernos locales durante el primer período legislativo democrático, 1979-1983, se puede argumentar que se dan características preexistentes de un sistema político totalitario. En los municipios pequeños del APIA, en ciertos casos los partidos políticos legalizados mantuvieron los alcaldes franquistas al frente de la lista de candidatos al gobierno local. Los cambios y evolución en el número de mujeres como miembros del consistorio a nivel municipal para cada una de las seis comarcas del APIA y para los 4 períodos democráticos legislativos: 1979-1983, 1991-1995, 2003-07 y 2007-11 se muestran en la tabla 4. 
Tabla 4

EVOLUCIÓN DE LAPRESENCIA DE MUJERES EN LOS AYUNTAMIENTOS

\begin{tabular}{|c|c|c|c|c|c|c|c|c|c|c|c|c|}
\hline \multirow[b]{2}{*}{ Comarcas } & \multicolumn{3}{|c|}{$1^{a}$ PL 1979-83 } & \multicolumn{3}{|c|}{$4^{\text {a }}$ PL 1991-95 } & \multicolumn{3}{|c|}{$7^{\mathrm{a}}$ PL 2003-07 } & \multicolumn{3}{|c|}{$8^{\text {a }}$ PL 2007-11 } \\
\hline & $\begin{array}{c}\mathbf{M} \\
(\mathbf{N})\end{array}$ & $\begin{array}{c}\text { M } \\
(\%)\end{array}$ & \begin{tabular}{|c} 
Total \\
(N)
\end{tabular} & $\begin{array}{c}\text { M } \\
\text { (N) }\end{array}$ & $\begin{array}{c}\text { M } \\
(\%)\end{array}$ & $\begin{array}{c}\text { Total } \\
\text { (N) }\end{array}$ & $\begin{array}{l}\mathbf{M} \\
(\mathbf{N})\end{array}$ & $\begin{array}{c}\mathbf{M} \\
(\%)\end{array}$ & \begin{tabular}{|c} 
Total \\
(N)
\end{tabular} & $\begin{array}{l}\mathbf{M} \\
(\mathbf{N})\end{array}$ & $\underset{(\%)}{\mathbf{M}}$ & $\begin{array}{l}\text { Total } \\
\text { (N) }\end{array}$ \\
\hline Alt Urgell & 1 & 0,8 & 131 & 8 & 6,4 & 125 & 21 & 17,5 & 120 & 26 & 20,6 & 126 \\
\hline Alta Ribagorça & 1 & 4,0 & 25 & 5 & 20,0 & 25 & 6 & 24,0 & 25 & 6 & 22,2 & 27 \\
\hline Cerdanya & 3 & 2,8 & 107 & 6 & 5,9 & 102 & 17 & 15,6 & 109 & 28 & 25,9 & 108 \\
\hline Pallars Jussà & 5 & 5,2 & 97 & 10 & 10,2 & 98 & 17 & 17,3 & 98 & 20 & 20,6 & 97 \\
\hline Pallars Sobirà & 2 & 2,1 & 97 & 11 & 12,4 & 89 & 21 & 23,1 & 91 & 21 & 21,4 & 98 \\
\hline Val d'Aran & 8 & 13,6 & 59 & 7 & 13,7 & 51 & 7 & 13,7 & 51 & 11 & 20,0 & 55 \\
\hline APIA & 20 & 3,9 & 516 & 47 & 9,6 & 490 & 89 & 18,0 & 494 & 112 & 21,9 & 511 \\
\hline Cataluña & 393 & 4,8 & 8262 & 919 & 11,0 & 8.321 & 2.014 & 23,2 & 8682 & 2.651 & 29,6 & 8953 \\
\hline
\end{tabular}

Nota: PL= Periodo Legislativo; $\mathrm{M}=$ Mujeres; $\mathrm{N}=$ total Hombres y Mujeres Fuente: Elaboración a partir de datos de Corcoy y Gómez, 2008.

Estos datos permiten establecer comparaciones entre las seis comarcas, y entre éstas y Cataluña. De media, las seis comarcas presentan una participación de la mujer consistentemente baja a lo largo de los períodos legislativos con respecto a la media catalana. En promedio, el APIA ha evolucionado a partir de una presencia femenina muy baja de 3,9\% para el primer período, a casi un $22 \%$ en un período de ocho legislaturas. En términos generales, podríamos afirmar que la presencia de las mujeres en los ayuntamientos ha seguido una tendencia general de mejora respecto a los nombramientos políticos, pero, en comparación con el conjunto de Cataluña, las comarcas de montaña han mostrado un ritmo más lento de adaptación a la nueva sociedad democrática.

Con el objetivo de analizar la mejora de la participación de las mujeres, nos centramos en el número de miembros del consistorio que son reelegidos. La tabla 5 indica para cada período legislativo si un miembro mujer del ayuntamiento fue elegida para este término y al menos otro, ya sea antes o después. Cataluña fluctúa entre el 3,5\% de reelegidas de la primera legislatura a un $21,7 \%$ de la octava. Por otra parte, en el APIA el porcentaje pasó de $2,7 \%$ a tan sólo el 14,3\%. Esta diferencia muestra que las mujeres tienen una representación política más esporádica que los hombres. Esto se detecta, tanto en Cataluña con 38,9\% de mujeres frente a 58,9\% de hombres; como en los Pirineos $34.8 \%$ y 58,6\% respectivamente.

Para completar nuestro conocimiento de la presencia en política de las mujeres en el gobierno local del APIA se ha analizado las diferentes responsabilidades de las mujeres agrupadas en nueve áreas (tabla 6), para el período legislativo octavo (2007-2011). En primer lugar, vemos que analizando el porcentaje de mujeres en la categoría de «sin responsabilidad», el número total de mujeres miembros es de 80 en el APIA, lo cual representa el 71,4\% del total de los políticos sin responsabilidad específica. En comparación a toda Cataluña, el número de mujeres en esta condición asciende a 4.605; siendo el porcentaje de mujeres de poco más de la mitad, 51,4\%. Los hombres controlan el grupo de carteras que se consideran 
Tabla 5

MIEMBROS REELEGIDOS POR PERIODO LEGISLATIVO Y SEXO

\begin{tabular}{|c|c|c|c|c|c|c|c|c|c|c|c|c|c|c|}
\hline \multirow[b]{2}{*}{ Comarcas } & \multicolumn{3}{|c|}{$1^{\text {a }} \mathrm{PL}^{1} 1979-83$} & \multicolumn{3}{|c|}{$4^{\mathrm{a}}$ PL 1991-95 } & \multicolumn{3}{|c|}{$7^{\mathrm{a}}$ PL 2003-07 } & \multicolumn{3}{|c|}{$8^{\text {a PL 2007-11 }}$} & \multirow{2}{*}{$\begin{array}{c}\% \mathrm{M} \\
\text { reelegidas } \\
\text { PL 2007-11 }\end{array}$} & \multirow{2}{*}{$\begin{array}{c}\% \mathrm{H} \\
\text { reelegidos } \\
\text { PL 2007-11 }\end{array}$} \\
\hline & $\begin{array}{l}\mathrm{M} \\
(\mathrm{N})\end{array}$ & $\begin{array}{c}\text { M } \\
(\%)\end{array}$ & $\begin{array}{c}\mathrm{T} \\
(\mathbf{N})\end{array}$ & $\begin{array}{c}\text { M } \\
(\mathrm{N})\end{array}$ & $\underset{(\%)}{M}$ & $\begin{array}{c}\mathrm{T} \\
(\mathrm{N})\end{array}$ & $\begin{array}{l}\text { M } \\
(\mathrm{N})\end{array}$ & $\begin{array}{c}M \\
(\%)\end{array}$ & $\begin{array}{c}\mathrm{T} \\
(\mathrm{N})\end{array}$ & $\begin{array}{c}\text { M } \\
(\mathrm{N})\end{array}$ & $\begin{array}{c}\text { M } \\
(\%)\end{array}$ & $\begin{array}{c}\mathrm{T} \\
(\mathbf{N})\end{array}$ & & \\
\hline Alt Urgell & 1 & 1,4 & 73 & 6 & 5,8 & 103 & 16 & 16,5 & 97 & 12 & 16.0 & 75 & 46.2 & 63.0 \\
\hline Alta Ribagorça & 1 & 12,5 & 8 & 3 & 17,6 & 17 & 5 & 31,3 & 16 & 2 & 18.2 & 11 & 33.3 & 42.9 \\
\hline Cerdanya & 0 & 0,0 & 53 & 3 & 3,6 & 83 & 10 & 11,5 & 87 & 7 & 12.1 & 58 & 25.0 & 63.8 \\
\hline Pallars Jussà & 2 & 4,5 & 44 & 6 & 7,6 & 79 & 5 & 10,6 & 47 & 5 & 9.6 & 52 & 25.0 & 61.0 \\
\hline Pallars Sobirà & 0 & 0,0 & 56 & 5 & 7,1 & 70 & 13 & 19,4 & 67 & 8 & 17.4 & 46 & 38.1 & 49.4 \\
\hline Val d'Aran & 3 & 12,0 & 25 & 3 & 7,3 & 41 & 7 & 15,6 & 45 & 5 & 16.1 & 31 & 45.5 & 59.1 \\
\hline APIA & 7 & 2,7 & 259 & 26 & 6,6 & 393 & 56 & 15,6 & 359 & 39 & 14.3 & 273 & 34.8 & 58.6 \\
\hline Cataluña & 124 & 3,5 & --- & 586 & 9,2 & --- & 136 & 20,7 & --- & 1 & 21.7 & --- & 38.9 & 58.9 \\
\hline
\end{tabular}

Nota: $\mathrm{PL}=$ Periodo Legislativo; $\mathrm{M}=$ Mujeres; $\mathrm{H}=$ Hombres; $\mathrm{N}=$ total Hombres y Mujeres

Fuente: Elaboración a partir de datos de Corcoy y Gómez, 2008.

Tabla 6

ÁREAS DE RESPONSABILIDAD DE LAS MUJERES (2007-2011)

\begin{tabular}{|l|c|c|c|c|c|c|}
\hline \multirow{2}{*}{\multicolumn{1}{|c}{ Responsabilidad política }} & \multicolumn{3}{|c|}{ APIA } & \multicolumn{3}{c|}{ Cataluña } \\
\cline { 2 - 8 } & $\begin{array}{c}\text { Mujeres } \\
(\mathbf{N})\end{array}$ & $\begin{array}{c}\text { Mujeres } \\
(\boldsymbol{\%})\end{array}$ & $\begin{array}{c}\text { Total } \\
(\mathbf{N})\end{array}$ & $\begin{array}{c}\text { Mujeres } \\
(\mathbf{N})\end{array}$ & $\begin{array}{c}\text { Mujeres } \\
(\boldsymbol{\%})\end{array}$ & $\begin{array}{c}\text { Total } \\
(\mathbf{N})\end{array}$ \\
\hline Administración e impuestos & 10 & 13,3 & 75 & 146 & 15,0 & 975 \\
\hline Infraestructuras y servicios municipales & 1 & 12,5 & 8 & 29 & 11,9 & 244 \\
\hline Participación ciudadanía y cooperación & 0 & - & 4 & 38 & 31,4 & 121 \\
\hline Servicios sociales & 16 & 29.1 & 55 & 724 & 43,0 & 1.682 \\
\hline Territorio y medio ambiente & 2 & 4,9 & 41 & 101 & 11,3 & 891 \\
\hline Comunicaciones y nuevas tecnologías & 0 & - & 0 & 5 & 25,0 & 20 \\
\hline Desarrollo económico & 3 & 17.6 & 17 & 83 & 20,0 & 415 \\
\hline Sin responsabilidad* & 80 & 25.7 & 311 & 1,525 & 33,1 & 4.605 \\
\hline \% sin responsabilidad/ Total & - & 71,4 & - & - & 51,4 & - \\
\hline Total & 112 & 21,9 & 511 & 2.651 & 29,6 & 8.953 \\
\hline
\end{tabular}

Nota: $\mathrm{N}=$ total Hombres y Mujeres; *son concejales y concejalas a los que no se les asigna ninguna responsabilidad concreta, pudiendo realizar los encargos que la alcaldía le haga en cada momento, o simplemente no tener responsabilidades al estar en la oposición.

Fuente: Elaboración a partir de datos de Corcoy y Gómez, 2008. 
políticamente más importantes, tales como: desarrollo económico (mujeres 20,0\% en Cataluña y el 17,6\% en APIA), administración e impuestos (15,0\% y 13,3 \%), infraestructuras y servicios municipales $(11,9 \%$ y $12,5 \%)$ o territorio y medio ambiente $(11,3 \%$ y $4,9 \%)$, incluido el desarrollo urbanístico. Encontramos la mayoría de las mujeres en servicios sociales, los cuales incluye educación, cultura y salud (43,0\% en Cataluña y 29,1\% APIA). Estos datos muestran que en general las mujeres están todavía marginadas en el acceso al poder local, un hecho que se manifiesta no sólo por su menor número sino también por las características de los cargos que ostentan.

\section{LA POLÍTICA RURAL DESDE EL DISCURSO DE SUS ACTORES}

Una vez identificadas el número de mujeres y las características de los cargos políticos que ostentan en el Pirineo catalán, a través del análisis temático de las entrevistas semiestructuradas realizadas a individuos de ambos sexos con responsabilidades políticas y/o técnicas, en este apartado se exploran los factores que dificultan y los que facilitan la participación de las mujeres en la política municipal.

\section{Factores que dificultan la participación de las mujeres}

Las mujeres en el ámbito rural sienten las obligaciones familiares, sea hacia la pareja, los hijos, los padres u otros familiares dependientes como prioritarias, y por tanto son activas en política en aquellos casos de menor necesidad de dedicación a la familia. Este es el caso de las mujeres que tienen los hijos mayores o padres con buena salud, al igual que las que cuentan con situaciones personales más independientes como por ejemplo el caso de mujeres solteras. El apoyo explícito de la pareja es importante. Esta problemática también la sufren las mujeres emprendedoras.

«De mujer que se cuida de su casa, de mujer que es el eje de la familia, que sí, que bien, que haga unos estudios y una carrerita, pero la familia es lo importante: aparcar tus necesidades personales exactamente. Yo no pensé que yo existía como tal, yo había existido en «función de», de mis padres primero, de mi madre, de mi marido, de mis hijos, de tal, y llega un momento en que el [nombre de un político local] me propone esto, y yo pienso «existo también». Pero fíjate el razonamiento, ahora que ya la familia más o menos, mis hijos ya son mayorcitos evidentemente, que tengo que estar a su lado, pero ya de otra manera, no me necesitan tan directamente. Quiero decir que ya hay una situación personal y familiar que me ayuda, pero si no, en ningún momento me lo había planteado yo eso.» (E-08-MP).

Las mujeres rurales muestran poco interés en la afiliación a partidos políticos y/o hacer carrera en el ámbito político. No perciben su participación como beneficiaria de intereses personales o profesionales, por el contrario, entienden la participación en la política como un acto de voluntad individual que implica sacrificios tanto en el ámbito personal como profesional. 
«No, militante no. Yo no era de ningún partido. [...] Porque me llamó la persona que había sido el alcalde anterior...» (E-22-MP).

«... Por estar en política [las mujeres] piensan que pueden incidir en la mejora del desarrollo local al estar en el Ayuntamiento ... con el objetivo de mejorar los servicios y las condiciones de vida de la población ... por tener más presente que los hombres las necesidades que hay en cada casa ...» (E-20-MT).

El control social de las mujeres en las áreas de montaña dificulta su participación en la vida pública ya que sienten más presión social que los hombres realizando la misma actividad.

«[...] una mujer quizá lo mira si eres criticado, si no eres criticado, me han dicho esto, me han dicho aquello. Quizás hay un poco más este punto flaco en mi opinión. Quizá sí que hay algunas cosas que frenan. Una mujer no tiene tanta facilidad» (E-29-HP).

«¿Sabes por qué? Yo te digo la verdad, el problema que había más grande era por las críticas. Vamos a ver cuando tú te metes en política, tú ya lo sabes, sobre todo en pueblos pequeños, se meten con tu vida, ¿me entiendes, que si tal, que si cual? Si a ti te importa mucho lo que lo demás dicen de ti en entonces ¿qué haces? Te reprochas y te quedas en casa y si tú dices yo porque me he de ocultar, yo no he hecho nada mal, yo quiero dedicarme y quiero hacer esto y además de una manera.» (E-22-MP).

«Y claro, esto es un pueblo, quieras o no quieras, nos conocemos todos, para ti políticamente significa dar un paso adelante y decir «Psst eh, [el nombre de la entrevistada] de toda la vida, ahora resulta que se define por un partido determinado» (E-08-MP).

«Mira, ponerte en política si vas de buena fe es ir a sufrir, y sí las mujeres esto quizás lo ven más que los hombres, porque los hombres se lo echan más a la espalda y si tú quieres ponerte en política para trabajar es ir a sufrir, porque nunca tendrás contento a todo el mundo, porque siempre hay quien quiere algo y hace mucho daño, y hace más una queja que diez alabanzas, y pienso que la mujer es más sensible y quizás lo ve más eso.» (E-6-MP).

El problema de las expectativas de rol de género también influye en minimizar las actividades de las mujeres como emprendedoras y políticas. La política local en alta montaña sigue aún vinculada a modelos patriarcales y actitudes caciquiles de defensa de intereses económicos particulares.

«A nivel de municipio y a nivel de comarca, yo creo que se funciona mucho con gente que son siempre los mismos los que mandan, aunque puedan cambiar 
de color político, o puedan cambiar de listas y tal, pero en realidad son siempre los mismos, ¿no? y yo creo que esto ha generado unas clientelas bastante enquistadas y bastante serias. Además hay otro componente y es que al haber tan poca gente, hay muchos lazos familiares, de intereses entre familias y entre grupos, y eso lo complica todo un poco.» (E-01-HP).

Aún existe una clara distinción entre la mujer en la esfera pública y en la esfera privada, lo cual promueve la invisibilidad de sus actuaciones en el ámbito económico. La figura del heredero persiste como elemento determinante en algunos pueblos pequeños del Pirineo:

«Es verdad que es una estructura de valle muy cerrado. Muy cerrada quiero decir, en el sentido conservador de la palabra. Donde la mujer tiene un papel bastante secundario. Donde las relaciones sociales están muy marcadas por una estructura tradicional ¿no? Aquí sí que el tema del heredero, por ejemplo existe, yo lo veo, más allá del valle» (E-13-MT).

«De alguna manera el rol masculino era buscarse la vida por ser el sostén de la familia, mientras que el rol de la mujer, era de alguna manera, casarse. Esto está cambiando, pero cuesta no tanto como nos pensamos, porque yo creo que este papel las chicas de hoy en día, yo tengo dos hijas eh! Y afortunadamente, no juegan este papel, quizá también porque han tenido la suerte de poder estudiar, de marchar, de irse a Barcelona muy pronto y tal, ¿no? Pero yo creo que la gente, que por las razones que sea, acaba quedando aquí arriba, sigue teniendo bastante este rol, bastante interiorizado. Y es difícil, ¿eh?» (E-03-HP).

A diferencia de los hombres en política, a menudo las mujeres no tienen conexiones previas ni familiares ni profesionales en el ámbito político. En ocasiones reúsan la inclusión en las listas, y su escasa presencia es interpretada como falta de aptitudes aunque se dan casos de participación femenina muy activa.

«Las mujeres, no hay ninguna mujer. Mi padre tenía una mujer en el equipo [...]. Hay mucha explicación para este momento. Había una mujer antes porque era una mujer adecuada. Porque era una mujer que estaba implicada y porque tenía ganas. Yo le propuse y en ese momento, ya no, ya no tenía ganas. Porque me gustaba su forma de hacer y realmente mirando el panorama pues no había. Básicamente, no es que no queramos, había mujeres capaces pero las mujeres capaces que me hubiera hecho ilusión que estuvieran en este momento no querían. No querían meterse en este mundo y por lo tanto, lo que nos quedaba eran o niñas o abuelas.» (E-01-HP).

«Me casé con un chico del Pallars y tengo dos hijos. Al decidirme a quedarme aquí, me vinieron a buscar para entrar en la lista porque somos pocos dispuestos y todos nos conocemos. He estado dos legislaturas en la oposición y una al gobierno» (E-24-MP). 
Hay una falta de comprensión y valoración por parte de los hombres activos en política en torno a las aportaciones que se pueden hacer desde una perspectiva de género. Los hombres activos en política caen en estereotipos de género.

«Yo creo, mi opinión al respecto, es que la Ley de la Paridad no tiene sentido para mí porque yo creo que si en un municipio hay cinco mujeres válidas, hay que ir cinco mujeres en la candidatura. Yo creo que tenemos que mirar más la validez de las personas $[\ldots . ..] \gg(\mathrm{E}-01-\mathrm{HP})$.

«Sí, a veces es interesante que haya una mujer para que por las fiestas del pueblo cuando tienes que organizar cosas para los niños tienen más idea que nosotros, cosas que pueda interesar a la montaña también. Sí, es muy importante para mí que haya mujeres y hay mujeres muy listas que da gusto verlas trabajar.» (E-29-HP).

\section{Factores que facilitan la participación de las mujeres}

La formación académica y los estudios fuera de las comarcas de montaña son factores que facilitan la participación de las mujeres en la política local.

«Cuando estudié fuera en Lleida y Barcelona, las juventudes de [nombre de un partido político] y [nombre de un partido político] hacían un frente común en contra de sus partidos [....] Hace unos quince años decidí volver porque había que hacer cosas por el Pallars y mejorar la sociedad.» (E-24-MP).

Las mujeres entran en política no tanto por iniciativa propia o familiar sino por requerimiento de formaciones políticas o individuos concretos que reconocen su valía y posible aportación dentro de las listas de sus partidos.

«El que ahora es el alcalde me comentó si quería formar parte de un equipo, que intentaba hacer algo nuevo con personas jóvenes y de cara a decir «venga», pongámonos a trabajar por el municipio, con un proyecto participativo que rompa esta forma de hacer, del alcalde por encima de todo, y claro que debe haber siempre quien marque, pero dando bastante juego a la participación y eso es lo que me convenció.» (E-05-MP).

«Me incorporé para hacer un servicio al municipio [...] y en el consejo comarcal hice lo mismo, más que política yo hacía un servicio a la comarca. Hacíamos reuniones semanales, los planes de comarca [...] hice lo que pude por mi gente y lo hice con mucho gusto.» (E-06-MP).

Las mujeres activas en política local provienen en algunos casos de contextos familiares desfavorecidos o experiencias personales difíciles dentro de sus unidades familiares, ante los cuales han mostrado un carácter combativo y luchador. Valoran la formación académica como herramienta de emancipación tanto social como económica. 
«Mira, vengo de familia trabajadora, a mí me habría gustado siempre estudiar, mucho, me encanta aún: conocer y saber [...] Y desde los 15 trabajé siempre los veranos para poder ganar algún dinero. Después, cuando fue la hora de continuar los estudios después de COU me pareció que Magisterio era la carrera más cortita, y los padres les parecía que podían llegar.» (E-08-MP).

La actividad política tiene como paso previo la activa vinculación a redes sociales y organizaciones cívicas. Las mujeres rurales activas en política tienen una visibilidad social previa y un sentido de compromiso y deber cívico con el municipio y la sociedad que las motiva a involucrarse en política como una extensión de su propuesta personal de servir a la colectividad.

«[...] Antes había sido mucho de movimientos asociativos, vengo del mundo asociativo.» (E-05-MP).

«[...] mira yo por ejemplo lunes y miércoles voy de voluntaria al [nombre del centro], sabes que me había ocupado siempre de la revista [nombre de la publicación] durante 32 años, ahora ya no, pero les sigo llevando la contabilidad, después cuando murió mi madre me dijo porque no nos ayudas con [nombre de organización]» (E-06-MP).

Las mujeres emprendedoras en actividades económicas y creación de nuevas empresas tienen más voluntad de participar activamente en la política local.

«Creo que las comunidades de montaña son bastante cerradas y por el hecho de ser mujer tienes que demostrar que vales mucho más que quizás un hombre y además en el sector [nombre de la actividad económica], que a veces hay parte de fuerza física, yo creo que el pueblo no acababan de entender que hacía... también creo que la sociedad avanza y acaba valorándose lo que es la persona. Además éramos tres chicas las que empezamos.» (E-05-MP).

La demanda de espacios para el turismo junto a la introducción de las TICs en las actividades productivas y en los servicios (Blanco, 2005) ha favorecido una mayor participación de las mujeres en la población activa, sea de forma visible o invisible. Los hoteles y las webs turísticas o de sectores como los artesanales han potenciado la combinación de actividades económicas en torno a la promoción turística.

«...yo soy también la presidenta de la Asociación de Artes y Oficios del Alt Pirineu i Aran. Cuando nació la asociación fue porqué el CEDRICAT realizó unos cursos de formación para los talleres artesanos, pero a base de ordenador, para que hiciéramos servir las nuevas tecnologías dentro de nuestro taller...» (E-14-DP)

La disponibilidad de tiempo vinculado al trabajo de media jornada o un trabajo en la administración pública, a menudo con horarios intensivos ayuda a conciliar el compromiso político. 
«Y las mujeres que aceptan nuestros planteamientos normalmente son mujeres que trabajan en la administración y que por tanto, quiere decir que tienen horarios o que pueden hacer la media jornada o que pueden trabajar en horarios más adecuados para la conciliación: jornada intensiva o en sectores como los hospitales.» (E-09-MP).

En el ejercicio de la actividad política las mujeres muestran un carácter más conciliador y de menor confrontación con voluntad de encontrar soluciones sin menoscabar los procedimientos. A la vez muestran autonomía y capacidad ejecutiva cuando consideran que han de tomarse medidas necesarias aunque impopulares.

«La mujer no busca, bueno, yo te digo mi opinión, conflictos pero en un momento determinado que has de tomar una decisión, cuando tú ves las cosas tú buscas soluciones y eres muy rápida, yo que me doy cuenta que junto a los hombres, o sea les damos cincuenta vueltas.» (E-22-MP).

«Las mujeres somos más sensibles, quizás porque vivimos más con el corazón que con la cabeza, somos más valientes, somos más atrevidas, y aunque el hombre diga que son del sexo fuerte, nosotras podemos tener miedo por muchas cosas, pero hay decisiones que un hombre no las haría nunca» (E-06-MP).

Las mujeres emigradas a las comarcas del APIA provenientes de otras partes del territorio con formaciones académicas, incorporadas a las administraciones locales como políticas o técnicas o con experiencia laboral fuera de la zona aportan nuevos valores e iniciativas en el ámbito local.

«Las mujeres del país controlan más desde la familia que desde el sector público. En cambio, las mujeres de fuera, técnicas, administración, neo-rurales son las que se han puesto más en política. Las mujeres tienen más agilidad en la administración y más eficiencia en la gestión. En cambio, los hombres están más metidos en obtener información y utilizarla y en controlar lo que se hace desde la administración y la sociedad en general.» (E-01-HP).

Las mujeres manifiestan más sensibilidad por temas sociales aunque, cuando tienen la oportunidad, desarrollan otras tareas con eficiencia dentro de la política rural. Hacen un buen uso de su inteligencia emocional.

«Yo creo que los temas más sociales se suelen dar a mujeres porque en aspectos relacionales y de consenso las mujeres solemos tenerlo más por mano, tenemos más experiencia. Y en cambio, la parte de obras es al revés, ya no te interesa tanto, a no ser que tengas una formación específica que haga que te interese el tema, la parte de obras es más dura para una mujer, en general, en cambio, prefieres creo a nivel de mujer pues eso, concejalías que tengan más trato humano, de dinamización social. «(E-05-MP). 


\section{REFLEXIONES FINALES}

La presente investigación analiza la participación política de las mujeres en territorios de alta montaña. La aproximación a la perspectiva de género parte de la visión de las mujeres como agentes necesarios de la dinamización económica y de la política local. El Alto Pirineo y Arán (APIA) es la región de Cataluña que presenta unas condiciones de orografía y clima más extremas. A pesar de las importantes mejoras en dotaciones tanto en servicios como en infraestructuras en los últimos años, y de producirse un importante avance en su sociedad civil, esta área rural presenta limitaciones con respecto a la participación de la mujer en la toma de decisiones. Los resultados de la presente investigación muestran que se ha producido una mejora en la incorporación de las mujeres a los órganos de representación política desde las primeras elecciones locales de 1979 hasta la fecha. Sin embargo, los datos también permiten observar que Cataluña se encuentra por debajo de la media de las comunidades autónomas en cuanto al número de mujeres en los parlamentos autonómicos y en las administraciones locales. En este contexto de inferioridad numérica, las comarcas Pirenaicas se muestran significativamente por debajo del resto de Cataluña.

En promedio, el APIA ha evolucionado a partir de una presencia femenina muy baja de $3,9 \%$ para el primer período electoral de $1979-83$, a casi un $22 \%$ en el periodo $2007-11$. En este sentido, en términos generales, podríamos afirmar que la presencia de las mujeres en los ayuntamientos ha seguido una tendencia general de mejora respecto a los nombramientos políticos, pero, en comparación con el conjunto de Cataluña, las comarcas de montaña han mostrado un ritmo más lento de adaptación a la nueva sociedad democrática. En el análisis de las diferentes responsabilidades, para el período 2007-2011 se observa que el porcentaje de mujeres en la categoría de «sin responsabilidad» equivale a un total de 60,9\% en el APIA, en comparación a toda Cataluña, que es del 51,4\%. Así mismo, los hombres controlan el grupo de carteras que se consideran políticamente más importantes, como son las de desarrollo económico (17,6\% en APIA), administración e impuestos $(13,3 \%)$, infraestructuras y servicios municipales $(12,5 \%)$ o territorio y medio ambiente $(4,9 \%)$, incluido el desarrollo urbanístico. Como se ha apuntado, estos datos muestran que en el ámbito de la política municipal las mujeres están todavía marginadas en el acceso al poder, un hecho que se manifiesta no sólo por su menor número sino también por las características de los cargos que ostentan.

En el estudio de la participación política y gobernanza local de las mujeres en las seis comarcas de la APIA, el análisis del material documental, bibliográfico y las entrevistas semi-estructuradas nos permiten identificar un grupo de factores que dificultan o limitan la participación de las mujeres en política local en el ámbito rural de la alta montaña. Con relación a los factores que se identifican como oferta, es decir, desde la actitud de las mujeres respecto a su voluntad de involucrarse en política observamos que las mujeres rurales sienten las obligaciones familiares como prioritarias, y las anteponen a su activismo político. Además muestran poco interés en la afiliación a partidos políticos y entienden la participación en la política como un acto que implica sacrificios personales y profesionales. El fuerte control social de las áreas de rurales también dificulta la participación de las mujeres en la vida pública. Así mismo, las expectativas de rol de género limitan las actividades de las mujeres, condicionando su participación y liderazgo tanto en calidad de emprendedoras económicas como en política local. También, se determina que muchas mujeres activas en política no 
tienen conexiones previas, ni familiares ni profesionales, en el ámbito político, contrariamente a como sucede a menudo con los hombres. Ello implica que las mujeres tienen que introducirse en círculos y actividades desconocidas, y que por ello sean aún más reticentes a la participación.

Con respecto a las limitaciones provenientes de la demanda, es decir, desde los propios partidos políticos, se observa que a menudo los hombres caen en estereotipos de género y valoran poco las aportaciones desde una perspectiva de género. Las leyes que obligan a la inclusión de mujeres en las listas electorales se identifican como factor muy positivo. De esta forma, la Ley de Igualdad aprobada en el 2007 ha sido crucial no sólo para introducir mujeres en los municipios de más de 5.000 habitantes (obligación que se extiende a los de 3.000 a partir del 2011), sino que también ha tenido su influencia en municipios más pequeños.

Finalmente, el análisis cualitativo también ha permitido identificar los factores que facilitan la participación de las mujeres rurales en política local. La formación académica, el haber estudiado o residido fuera de las comarcas de montaña o la procedencia de otros lugares son elementos incentivadores. La disponibilidad de tiempo, ya sea con un trabajo de media jornada o con un trabajo en la administración de horario intensivo ayuda a conciliar y posibilita una responsabilidad política. Así mismo se ha podido establecer que las mujeres entran en política por requerimiento de formaciones políticas o individuos concretos, más que por iniciativa propia o familiar. La motivación principal es el servicio a la comunidad. Son mujeres que tienen una visibilidad social previa y un sentido de compromiso y deber cívico con el municipio y la sociedad rural. A menudo han formado parte de organizaciones cívicas, a las que retornan una vez abandonan la política. Las mujeres emprendedoras en actividades económicas también tienen más voluntad de participar activamente en la política local. Futura investigación debería aportar nuevos datos a cerca de esta línea de análisis.

\section{BIBLIOGRAFÍA}

BENERÍA, L. y SEN, G. (1982): «Class and Gender Inequalities and Women's Role in Economic Development: Theoretical and Practical Implications». Feminist Studies 8(1): 157-176.

BINIMELIS, R.; BOSCH, M.; HERRERO, A. (2008): A sol i serena: dones, món rural $i$ pagesia. Barcelona. Institut Català de la Dona.

BLANCO ROMERO, A. y CÀNOVES VALIENTE, G. (2005): «Las tecnologías de la información y la comunicación en el desarrollo del turismo rural» en Documents d'Anàlisi Geogràfica, 46, 105-117.

BRABO, Tânia (2008): Gênero e Poder Local, Humanitas, San Pablo.

CASELlAS, A., TULLA, A.F. y PALLARÈS-BLANCH, M. (2009): «Women's political participation in Parliamentary Democracy in Rural Catalonia (1970's - 2000's), Analele Universităţii de Vest din Timişoara, GEOGRAFIE, vol. XIX, 11-26.

CORCOY RIUS, M. y LAURA GÓMEZ, P.L. (2008): Elaboració de la Base de Dades: Dones $i$ homes Protagonistes a les Institucions Democràtiques Catalanes. Barcelona. Institut de Ciències Polítiques i Socials.

DEPARTAMENT DE TERRITORI I SOSTENIBILITAT. MEDI AMBIENT. GENERALITAT DE CATALUNYA (2011): Bases cartogràfiques. http://www.gencat.cat (última consulta 25/05/2012). 
FARINÓS DASÍ, J. (2008): «Gobernanza territorial para el desarrollo sostenible: estado de la cuestión y agenda» Boletín de la Asociación de Geógrafos Españoles, vol. 46, 11-32.

GARCIA-RAMON, M.D.; CÀNOVES, G. y VALDOVINOS, N. (1995): «Farm tourism, gender and the environment in Spain». Annals of Tourism Research, 22 (2), 267-282.

GUIRADO, C. (2011): Tornant a la muntanya. Migració, ruralitat i canvi social al Pirineu català. El cas del Pallars Sobirà. Tesi de Doctorat, Departament de Geografia UAB.

HARLEY, S. Ed. (2007): Women's Labor in the Global Economy: Speaking in Multiple Voices. New Brunswick, NJ. Rutgers University Press.

INSTITUT D'ESTADÍSTICA DE CATALUNYA (IDESCAT) (2011a): Bases de dades territorials. Estadístiques de la població, 1900-2010. http://www.idescat.net

INSTITUT D'ESTADÍSTICA DE CATALUNYA (IDESCAT) (2011b): Anuari estadístic de Catalunya 2010. http://www.idescat.net

INSTITUTO DE LA MUJER (2010a): Base de datos sobre Parlamentos Autonómicos por CC.AA. http://www.inmujer.migualdad.es/mujer/mujeres/cifras/poder/poder_ejecutivo. htm, (última consulta 1/10/2011).

INSTITUTO DE LA MUJER (2010b): Base de datos sobre Alcaldías por CC.AA. http:// www.inmujer.migualdad.es/mujer/mujeres/cifras/poder/poder_ejecutivo.htm, (última consulta $1 / 10 / 2011)$.

INSTITUTO DE LA MUJER (2010c): Base de datos sobre Concejalas por CC.AA. http:// www.inmujer.migualdad.es/mujer/mujeres/cifras/poder/poder_ejecutivo.htm, (última consulta $1 / 10 / 2011)$.

LEININGER, M. M. (1985): Qualitative research methods in nursing (pp. 33-72). Orlando. Grune \& Stratton.

MALINOWSKI, B. (1955): Sex and Repression in Savage Society. New York. Meridian.

MASSOLO, A. (2003): «Participación de las mujeres en los gobiernos locales de América Latina», en BARRERA, D. \& MASSOLO, A. (Coords.): Memoria del Primer Encuentro Nacional de Presidentas Municipales, Instituto Nacional de Mujeres, México, 1-19.

MINISTERIO DE MEDIO AMBIENTE, RURAL Y MARINO (2011): Diagnóstico de la igualdad de género en el mundo rural. Madrid. MARM.

MONK, S. y HODGE, I. (1995): «Labour market and employment opportunities in rural Britain». Sociologia Ruralis, 2, Vol. XXXV, 153-172.

NOGUÉ, J. (1988): «El fenómeno neorural». Agricultura y Sociedad, 47: 145-175.

NORRIS, P. (1997): Passages to Power. Legislative recruitment in advanced democracies. Cambridge. Cambridge University Press.

OTERO, I. y LOIS, M. (2006): «La presencia de las mujeres en las comunidades autónomas ¿Algo más que meras intenciones?» en HURTADO SÁNCHEZ, J. y VVAA, La mujer como sujeto de la acción política, Centro de Estudios Andaluces, Consejería de la Presidencia e Igualdad, Sevilla, 91-143.

PALENZUELO CHAMORRO, P.; JORDI SÁNCHEZ, M. y CRUCES ROLDÁN, C. (2002): Mujeres Empresarias y Mujeres Políticas en el Medio Rural Andaluz, Universidad de Sevilla y Consejería de Agricultura y Pesca, Sevilla.

PUJOL, H. y ORTIZ, A. (2009): «Acadèmia i igualtat de gènere: només un miratge?». Documents d'Anàlisi Geogràfica, 55, 129-145. 
SILVA, M. (2011): «Geografia e gênero em assentamentos rurais: espaços de poder» en SILVA, J. \& PINHEIRO, C. (Eds.): Espaço, gênero e poder: conectando fronteiras, Todapalavra, Ponta Grossa (Parana, Brasil), 137-147.

SORIANO, J. M. y TULLA, A. F. (2003): «El repoblament del Pirineu Català: desig o realitat?». Metode. Revista de Difusió de la Investigació de la Universitat de Valencia, 36, 65-70.

SPRADLEY, J. (1979): The ethnographic interview. New York. Holt, Rinehart and Winston.

TAYLOR S. J. y BOARD, R. (1984): Introduction to qualitative research methods: The search for meanings. New York. John Wiley \& Sons.

TIMAR, J. (2004): «Gendered urban policy-making: the role of geographical scale in women's participation in Hungarian local governments, en CORTESI, G.; CRISTALDI, F. \& DROOGLEEVER, F. (Eds.): Gendered Cities, Identities, activities, networks. A lifecourse approach, Societa Geografica Italiana, Roma, 227-243.

TULLA, A.F. (1991): «Women and family farms in Catalonia». Iberian Studies, 20 (1i2): $62-80$.

UNDP (2003): Gender and Governance. India: Human Development Resource Centre \& United Nations Development Programme.

VALCÁRCEL, A. (1997): La política de las mujeres. Madrid. Càtedra.

VERGE, T. (2010): «Gendering representation in Spain: opportunities and limits of gender quotas» Journal of Women, Politics and Policy. 31(2), 166-190.

VILADOMIU, L. y ROSELL, J. (1998): «Evaluando políticas, programas y actuaciones de desarrollo rural». Revista española de economía agraria, 182, 297-308.

VILAGRASA, J. (2003): «L'Alt Pirineu i Aran, entre l'eficiència econòmica i la sostenibilitat». Papers, Regió Metropolitana de Barcelona, 39, 127-149.

WOLLSTONECRAFT, M. (1975): Vindication of the Rights of Women. London, Penguin. 